\title{
Prevalência das famílias TEM, SHV e CTX-M de $\beta$-lactamases de espectro estendido em Escherichia coli e Klebsiella spp no Hospital Universitário de Santa Maria, Estado do Rio Grande do Sul
}

\author{
Prevalence of the TEM, SHV and CTX-M families of extended-spectrum \\ $\beta$-lactamases in Escherichia coli and Klebsiella spp at the \\ University Hospital of Santa Maria, State of Rio Grande do Sul
}

\author{
Caio Fernando de Oliveira ${ }^{1}$, Nara Lucia Frazzon Dal Forno ${ }^{2}$, Izabel Almeida Alves ${ }^{1}$, \\ Jorge André Horta ${ }^{3,4}$, Alexandre Rieger ${ }^{3,4}$ e Sydney Hartz Alves ${ }^{5}$
}

\begin{abstract}
RESUMO
Neste estudo estimou-se a distribuição e prevalência de $\beta$-lactamases de espectro estendido pertencentes às famílias TEM, SHV e CTX-M entre amostras de Escherichia coli e Klebsiella spp. no Hospital Universitário de Santa Maria, Rio Grande do Sul. Durante 14 meses, 90 microrganismos foram selecionados como prováveis produtores de ESBL. Os isolados foram submetidos a testes fenotípicos confirmatórios para a presença de ESBL. A seguir, os tipos de ESBLs presentes em cada microrganismo foram determinados através da pesquisa dos respectivos genes através da reação em cadeia da polimerase. Empregando-se o método do disco combinado, a presença de ESBLs foi confirmada em 55 (61,1\%) amostras; quando o método do duplo disco foi utilizado, 57 (63,3\%) amostras foram produtoras de ESBLs. Com base na PCR, as ESBLs do tipo TEM e SHV foram mais presentes em Klebsiella pneumoniae enquanto que ESBL do tipo CTX-M foram mais presentes em Klebsiella oxytoca.
\end{abstract}

Palavras-chaves: $\beta$-lactamases de espectro estendido. Escherichia coli. Klebsiella spp. Reação em cadeia da polimerase.

\begin{abstract}
In this study, the distribution and prevalence of extended-spectrum $\beta$-lactamases belonging to the TEM, SHV and CTX-M families were estimated among samples of Escherichia coli and Klebsiella spp. at the university hospital of Santa Maria, Rio Grande do Sul. Over a 14-month period, 90 microorganisms were selected as likely ESBL producers. The isolates were subjected to confirmatory phenotype tests for the presence of ESBL. Through investigating the respective genes using the polymerase chain reaction, the ESBL types present in each microorganism were then determined. Fifty-five samples (61.1\%) were confirmed as ESBL-positive by means of the combined disc method, and 57 (63.3\%) were found to be ESBL producers by means of the double disc method. From the polymerase chain reaction, ESBLs of TEM and SHV types were more frequently present in Klebsiella pneumoniae, while ESBL of CTX-M type was more frequently present in Klebsiella oxytoca.
\end{abstract}

Key-words: extended-spectrum $\beta$-lactamases. Escherichia coli. Klebsiella spp. polymerase chain reaction.

As $\beta$-lactamases de espectro estendido (ESBL) são enzimas capazes de hidrolisar penicilinas, cefalosporinas de primeira, segunda, terceira e quarta gerações e o monobactâmico aztreonam, mas não as cefamicinas e os carbapenens. Entretanto,

1. Curso de Pós-Graduação em Ciências Farmacêuticas, Universidade Federal de Santa Maria, Santa Maria, RS. 2. Hospital Universitário de Santa Maria, Universidade Federal de Santa Maria, Santa Maria, RS. 3. Departamento de Biologia e Farmácia, Universidade de Santa Cruz do Sul, Santa Cruz do Sul, RS. 4. Laboratório de Biotecnologia e Genética, Universidade de Santa Cruz do Sul, Santa Cruz do Sul, RS. 5. Departamento de Microbiologia e Parasitologia, Universidade Federal de Santa Maria, Santa Maria, RS.

Endereço para correspondência: Prof. Sydney Hartz Alves. Laboratório de Pesquisas Micológicas/Depto. de Microbiologia e Parasitologia/UFSM. Prédio 20, sala 4139, Campus da UFSM, 97105-900 Santa Maria, RS.

Telefax: $55553220-8906$

e-mail: hartzsa@smail.ufsm.br

Recebido para publicação em 09/06/2009

Aceito em 14/08/2009 são inibidas por inibidores de $\beta$-lactamases como ácido clavulânico, sulbactam e tazobactam. Esta propriedade é a base dos testes laboratoriais utilizados para a detecção in vitro dessas enzimas $^{17}{ }^{18}$. Klebsiella pneumoniae e Escherichia coli são as espécies mais comumente relacionadas como produtoras de ESBLS $^{817}$.

A maioria das ESBLs evoluiu a partir de mutações genéticas em $\beta$-lactamases clássicas (TEM-1, TEM-2 e SHV-1), originando principalmente variedades de ESBLs dos tipos TEM e SHV ${ }^{17}$. Contudo, uma nova família de ESBLs, a CTX-M, emergiu nos últimos anos, principalmente em Escherichia coli, e tem se tornado uma das mais prevalentes famílias dessas enzimas em muitos países ${ }^{211} 141821$.

A presença de ESBLs nos ambientes hospitalares e suas consequiências nas estratégias de tratamento com antimicrobianos constitui um importante agravante para pacientes hospitalizados ${ }^{20}$. 
O monitoramento da prevalência e dos tipos de ESBLs é fundamental para o melhor entendimento desse problema e para a definição de apropriadas opções terapêuticas ${ }^{12}$. No Brasil, embora isolados clínicos produtores de ESBL tenham sido ocasionalmente relatados, ${ }^{81626}$ estudos com relação a prevalência das famílias de ESBL merecem ser abordados.

0 teste fenotípico de disco-difusão é a principal metodologia utilizada atualmente nos laboratórios brasileiros para a detecção de ESBLs. Entretanto, a detecção baseada nestes testes já foi alvo de diversos estudos ${ }^{4222530}$ onde foi estabelecido que mecanismos adicionais de resistência (como $\beta$-lactamases do tipo AmpC) podem levar a resultados divergentes ${ }^{1928} 30$.

Neste estudo, avaliou-se, através de dois testes confirmatórios fenotípicos e um molecular, a prevalência de ESBLs durante catorze meses em um hospital universitário de referência regional no centro do Estado do Rio Grande do Sul. Além disso, avaliouse a distribuição das ESBLs entre os isolados através da PCR. Também foi pesquisada a possível presença de outros mecanismos de resistência que possam prejudicar a detecção de ESBLs por métodos fenotípicos.

\section{MATERIAL E MÉTODOS}

Seleção dos microrganismos. Entre abril de 2007 e maio de 2008, foram selecionados 90 isolados clínicos consecutivos não-duplicados de Escherichia coli e Klebsiella spp provenientes de materiais biológicos diversos (ferida operatória, escara, urina, swab anal, secreção traqueal, escarro, etc) de pacientes internados no Hospital Universitário de Santa Maria (HUSM). Os microrganismos foram identificados através do sistema automatizado autoSCAN-4 (Dade Behring Inc, West Sacramento, CA, USA) de acordo com os critérios do $\mathrm{CLSI}^{6}$ para prováveis produtores de ESBLs e foram armazenados em glicerol a $-20^{\circ} \mathrm{C}$.

Testes fenotípicos confirmatórios para $\beta$-lactamases de espectro estendido. Teste do disco combinado: foram utilizados os seguintes discos: cefpodoxima $(10 \mu \mathrm{g})$, ceftazidima $(30 \mu \mathrm{g})$ e cefotaxima $(30 \mu \mathrm{g})$ isoladamente e em associação com

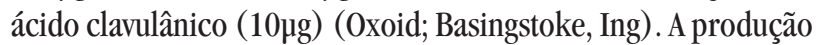
de ESBL foi verificada pelo aumento $(\geq 5 \mathrm{~mm})$ no diâmetro do halo de inibição dos discos contendo o antibiótico em combinação com o ácido clavulânico se comparados ao mesmo antibacteriano sem a presença deste inibidor.

Teste sinérgico do duplo disco: os mesmos discos de antibacterianos citados anteriormente (com exceção ao disco de ácido clavulânico) foram distribuídos a uma distância de $20 \mathrm{~mm}$ de um disco contendo amoxicilina/ácido clavulânico (20/10 $\mu$ g) (Oxoid; Basingstoke, Ing). Qualquer aumento da zona de inibição de um dos antibióticos em direção ao disco de amoxicilina + ácido clavulânico era indicativo da produção de ESBL. Ambos os testes fenotípicos foram realizados em ágar Mueller-Hinton (Merck; Darmstadt, Germany) e interpretados de acordo com as recomendações do CLSI $^{56}$ para testes de disco difusão em ágar.

Teste de susceptibilidade à cefoxitina. Os microrganismos que evidenciaram ausência de ESBLs em qualquer um dos métodos fenotípicos foram avaliados frente a cefoxitina (30 $\mu \mathrm{g})$ (Oxoid; Basingstoke, Ing). A resistência à cefoxitina está associada a presença de outros mecanismos de resistência que podem interferir na detecção de ESBLs pelos métodos fenotípicos ${ }^{28}$.

Caracterização molecular dos tipos de $\beta$-lactamases de espectro estendido. Extração de DNA: a extração do DNA foi realizada de acordo com a metodologia descrita por van Soolingen ${ }^{31}$. Na etapa inicial, com o objetivo de obter maior quantidade de DNA, cada microrganismo foi inoculado em $2 \mathrm{~mL}$ de caldo BHI (Merck; Darmstadt, Germany) e incubado a $37^{\circ} \mathrm{C}$ por 24 horas.

Reação em cadeia da polimerase: foi realizada para confirmar a presença dos genes codificadores de ESBLs dos tipos TEM, SHV e CTX-M em cada isolado. 0 par de primers 5'- ATG AGT ATT CAA CAT TTC CG -3' e 5'- CTG ACA GTT ACC AAT GCT TA $-3^{23}$ foi utilizado para amplificação de uma sequência de 1.076 pares de bases da família TEM. Para a família SVH foi utilizado o par de primers 5'- TTA GCG TTG CCA GTG CTC -3' e 5'- GGG TTA TTC TTA TTT GTC GC -3²3 (930pb) e para a família CTX-M, 5'- ACC GCG ATA TCG TTG GT -3' e 5'- CGC TTT GCG ATG TGC AG $-3^{3}(550 \mathrm{pb})$. Os primers foram comprados de Integrated DNA Technologies, Inc. (Coralville, USA). Todas as reações foram realizadas com $1 \mu \mathrm{L}$ do DNA de cada microrganismo, primers específicos, 1U de Taq DNA polimerase (Ludwig Biotec), tampão $\mathrm{MgCl}_{2}$ (Fermentas), dNTP (Ludwig Biotec) e água miliQ em um volume total de $25 \mu \mathrm{L}$. As condições das reações incluíram 5 min de desnaturação a $94^{\circ} \mathrm{C}\left(95^{\circ} \mathrm{C}\right.$ para SHV e 7 min para CTX-M) seguidos de 35 ciclos (30 para TEM) de: desnaturação $\left(94^{\circ} \mathrm{C}\right.$ por $30 \mathrm{~s}$ para TEM e $60 \mathrm{~s}$ para CTX-M e $95^{\circ} \mathrm{C}$ por $45 \mathrm{~s}$ para SHV), anelamento $\left(58^{\circ} \mathrm{C}\right.$ por 1 min para TEM, $59^{\circ} \mathrm{C}$ por $45 \mathrm{~s}$ para SHV e $54^{\circ} \mathrm{C}$ por $45 \mathrm{~s}$ para CTX-M) e extensão $\left(72^{\circ} \mathrm{C}\right.$ por 1 min), finalizando com um período de extensão final de $72^{\circ} \mathrm{C}$ por $7 \mathrm{~min}$. As amplificações foram realizadas no termociclador MJ96+/MJ96G (Biocycler).

Avaliação do padrão dos amplicons de DNA: o produto amplificado de cada reação foi submetido à eletroforese em gel agarose $1 \%$ (BioRad) com brometo de etídio $(0,5 \mu \mathrm{g} / \mathrm{mL})$ durante 1 hora (6 volts/cm) e visualizado em UV (312nm) com auxílio do Transluminador Série-ECX (Vilber Lourmat) sendo foto documentado pelo sistema DP-001.FDC (Vilber Lourmat). Os microrganismos com genes para uma determinada família de ESBL evidenciaram o mesmo tamanho de DNA que a sequência iniciadora amplifica o que foi confirmado pelo controle positivo e pelo marcador de tamanho molecular (Figura 1).

Microrganismos controle: Klebsiella pneumoniae ATCC 700603 (bla $\left.{ }_{\mathrm{SHV}-18}\right)$, Escherichia coli ATCC 25922 e Escherichia coli VA1341/03 (CTX-M-1) ${ }^{13}$.

\section{RESULTADOS}

Na triagem inicial, os 90 microrganismos selecionados como prováveis produtores de ESBL foram identificados como Klebsiella pneumoniae $\left(\mathrm{n}^{\mathrm{0}}=64\right)$, Escherichia coli $\left(\mathrm{n}^{\mathrm{0}}=22\right)$ e Klebsiella oxytoca $\left(\mathrm{n}^{\mathrm{0}}=4\right)$ correspondendo, respectivamente, a $71,1 \%$, $24,4 \%$ e $4,4 \%$ das amostras deste estudo. 


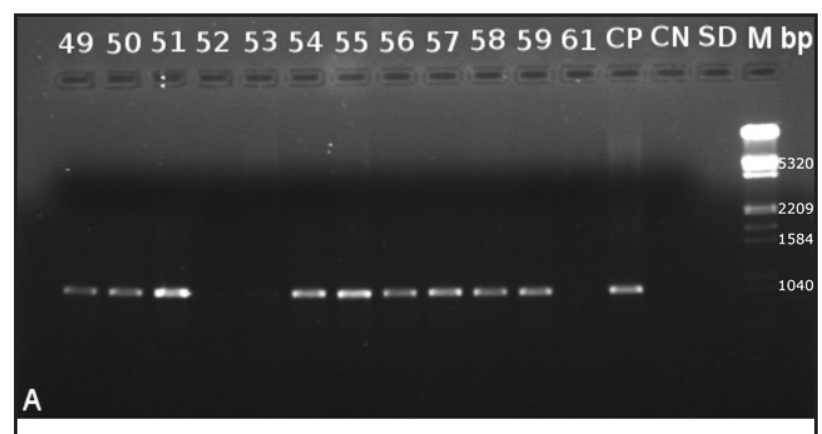

$\begin{array}{llllllllllllllll}23 & 24 & 25 & 26 & 27 & 28 & 30 & 31 & 32 & 33 & 34 & 35 & 36 & 37 & 38 & M \\ \text { bp }\end{array}$

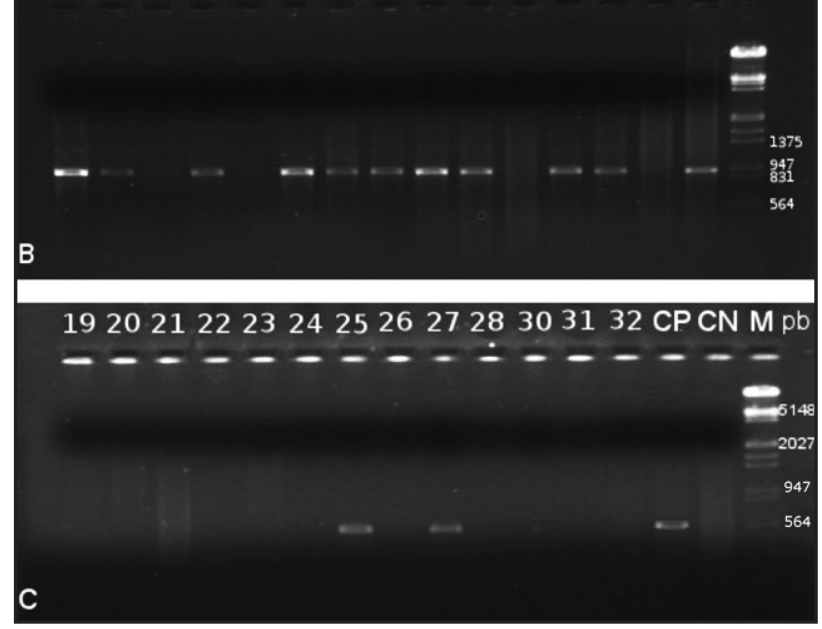

CP: controle positivo, CN: controle negativo, SD: sem DNA, M: marcador de tamanho molecular de DNA (PB-L Productos Bio-Lógicos) em pares de bases (pb).

\section{FIGURA 1}

Visualização do produto amplificado em gel de agarose. A: PCR com primers para ESBLs do tipo TEM, B: SHV, C: CTX-M.

Em relação ao desempenho dos testes fenotípicos confirmatórios, em 67,8\% (61/90) das amostras selecionadas foi observado sinergismo entre o ácido clavulânico e pelo menos uma das cefalosporinas testadas pelos métodos fenotípicos utilizados. 0 teste sinérgico do duplo disco confirmou 57 (63,3\%) isolados e o teste do disco combinado 55 (61,1\%) dos microrganismos, como produtores de ESBLs (Tabela 1). Da mesma forma, analisando esses resultados divididos entre as espécies, estes testes confirmaram 46 e 45 Klebsiella pneumoniae, 9 e 8 Escherichia coli e ambos 2 Klebsiella oxytoca como microrganismos produtores de ESBLs, respectivamente, para duplo disco e disco combinado.

Com relação aos três substratos utilizados nos testes fenotípicos, houve diferenças em termos de sensibilidade de detecção quando cada substrato foi comparado com a detecção total do teste. A ceftazidima apresentou sensibilidade de $91,2 \%$ pelo duplo disco e 49,1\% pelo disco combinado; cefpodoxima evidenciou 87,3\% de sensibilidade pelo disco combinado e $63,2 \%$ pelo duplo disco. Já a cefotaxima demonstrou sensibilidades de 91,2\% e 81,8\% respectivamente, para duplo disco e disco combinado.

Quando se avaliou a susceptibilidade a cefoxitina, entre as 39 amostras testadas, 19 foram sensíveis, 16 resistentes e 4 foram consideradas intermediárias.

Os genes codificadores de ESBLs do tipo TEM foram detectados em $82,2 \%\left(n^{0}=74\right)$ das amostras, os do tipo SHV em $67,8 \%\left(n^{0}=61\right)$ e, do tipo CTX-M em 21,1\% ( $\left.n^{0}=19\right)$. Entre as espécies estudadas, Klebsiella pneumoniae foi a que evidenciou os maiores percentuais dos genes TEM $(89,1 \%)$ e SHV (79,7\%) enquanto em Klebsiella oxytoca o gene CTX-M foi detectado em $50 \%$ das cepas. Apenas um isolado de Escherichia coli evidenciou genes codificadores da família CTX-M (Tabela 2).

Quanto a presença de mais de um tipo de ESBL em uma mesma amostra, 39 isolados possuíam genes dos tipos TEM e SHV, 3 possuíam genes dos tipos TEM e CTX-M, e em apenas 1 isolado detectou-se a combinação dos genes SHV e CTX-M. Em 12 isolados foram detectados genes codificadores para os três tipos de ESBLs.

A presença de genes codificadores de pelo menos uma das três famílias de ESBLs foi constatada em 93,3\% (84/90) dos microrganismos selecionados assim distribuídos: Klebsiella oxytoca 100\% (4/4); Klebsiella pneumoniae 96,9\% (62/64) e Escherichia coli $81,8 \%$ (18/22) (Tabela 2).

TABELA 1

Comparação entre métodos fenotípicos para detecção de ESBLs destacando-se o desempenho individual de cada substrato.

\begin{tabular}{|c|c|c|c|c|c|c|c|c|}
\hline \multirow[b]{2}{*}{ Metodologia } & \multicolumn{2}{|c|}{ Ceftazidima } & \multicolumn{2}{|c|}{ Cefotaxima } & \multicolumn{2}{|c|}{ Cefpodoxima } & \multicolumn{2}{|c|}{ Total } \\
\hline & $\mathrm{n}^{\mathrm{o}}$ & $\%$ & $\mathrm{n}^{\mathrm{o}}$ & $\%$ & $\mathrm{n}^{\underline{0}}$ & $\%$ & $\mathrm{n}^{\mathrm{o}}$ & $\%$ \\
\hline Duplo disco & 52 & 91,2 & 52 & 91,2 & 36 & 63,2 & 57 & 100 \\
\hline Disco combinado & 27 & 49,1 & 45 & 81,8 & 48 & 87,3 & 55 & 100 \\
\hline Total & 57 & 93,4 & 59 & 96,7 & 53 & 86,9 & 61 & 100 \\
\hline
\end{tabular}

TABELA 2

Detecção dos genes codificadores das principais famílias de ESBLs em Escherichia coli e Klebsiella spp através da reação de PCR.

\begin{tabular}{|c|c|c|c|c|c|c|c|c|c|c|}
\hline \multirow[t]{3}{*}{ Microrganismos } & \multirow{2}{*}{\multicolumn{2}{|c|}{ TEM }} & \multirow{2}{*}{\multicolumn{2}{|c|}{ SHV }} & \multicolumn{6}{|c|}{ TEM ou SHV } \\
\hline & & & & & \multicolumn{2}{|c|}{ CTX-M } & \multicolumn{2}{|c|}{ ou CTX-M } & \multicolumn{2}{|c|}{ Total } \\
\hline & $\mathrm{n}^{0}$ & $\%$ & $\mathrm{n}^{0}$ & $\%$ & $\mathrm{n}^{0}$ & $\%$ & $\mathrm{n}^{0}$ & $\%$ & $\mathrm{n}^{0}$ & $\%$ \\
\hline Escherichia coli & 14 & 63,6 & 9 & 40,9 & 1 & 4,5 & 18 & 81,8 & 22 & 100 \\
\hline Klebsiella pneumoniae & 57 & 89,1 & 51 & 79,7 & 16 & 25 & 62 & 96,9 & 64 & 100 \\
\hline Klebsiella oxytoca & 3 & 75 & 1 & 25 & 2 & 50 & 4 & 100 & 4 & 100 \\
\hline Total & 74 & 82,2 & 61 & 67,8 & 19 & 21,1 & 84 & 93,3 & 90 & 100 \\
\hline
\end{tabular}




\section{DISCUSSÃo}

A prevalência de espécies bacterianas produtoras de ESBLs varia conforme o país ou região de abrangência do estudo, todavia, Klebsiella pneumoniae e Escherichia coli são as espécies mais frequentemente encontradas relacionadas a estas enzimas $^{891216192527}$. A espécie mais prevalente em nosso estudo e que apresentou maior quantidade de resultados positivos nos testes para a produção de ESBLs foi Klebsiella pneumoniae.

Os métodos do disco combinado e do duplo disco confirmaram a presença de ESBLs em respectivamente 61,1 e 63,3\% dos microrganismos selecionados (Tabela 2). No Brasil, Nogueira e cols ${ }^{16}$ confirmaram a presença de ESBLs pelo método do disco combinado em $78 \%$ de isolados da família Enterobacteriaceae previamente resistentes às cefalosporinas de terceira geração e ao aztreonam. No mesmo sentido, Freitas e cols ${ }^{8}$ utilizando o método do duplo disco obtiveram $26 \%$ de positividade avaliando amostras de Klebsiella spp e Escherichia coli previamente selecionadas como prováveis produtoras de ESBL. Em outros países a prevalência de ESBLs pelos métodos fenotípicos varia entre 20 a $67 \%{ }^{11} 1525293032$

Embora os resultados referentes a detecção de ESBLs pelos dois métodos fenotípicos tenham apresentado resultados semelhantes (61,1 e 63,3\%), nas 16 amostras com resultados discordantes foram evidenciadas diferenças entre as duas metodologias. A variação no desempenho de acordo com a distância entre os discos no método do duplo disco ${ }^{1730}$ e o desempenho individual de cada substrato nos dois métodos são fatores que podem ter contribuído para essa discordância ${ }^{22} 30$.

Pelo método do duplo disco, ceftazidima e cefotaxima permitiram ótimos índices de detecção de ESBLs. Já no método do disco combinado, cefpodoxima e cefotaxima constituíramse em bons substratos, mas a ceftazidima evidenciou baixo rendimento (Tabela 2 ). Com base no método do disco combinado, alguns estudos confirmam o baixo desempenho da ceftazidima principalmente na presença de ESBLs do tipo CTX-M ${ }^{22}{ }^{30}$. Contrariamente, Steward e cols ${ }^{28}$ relataram melhor desempenho da ceftazidima em relação à cefotaxima entre isolados de Klebsiella pneumoniae provenientes dos Estados Unidos. No Brasil, Nogueira e cols ${ }^{16}$ comparando substratos para a detecção de ESBLs relataram melhor desempenho da cefotaxima e menor eficiência da cefpodoxima e ceftazidima.

$\mathrm{Na}$ detecção molecular dos genes codificadores das enzimas ESBL, a técnica de PCR revelou genes de pelo menos uma das três famílias de ESBLs pesquisadas em 15 dos 16 isolados resistentes a cefoxitina. Tal achado é importante porque a resistência a cefoxitina foi correlacionada com a possível presença de outros mecanismos de resistência que podem prejudicar a detecção de ESBLs pelos métodos fenotípicos ${ }^{28}$.

Em concordância com outros estudos ${ }^{12} 15192527$ a grande maioria (82,2 e 67,8\%) dos nossos isolados evidenciou genes codificadores para os tipos TEM e SHV de ESBLs. A frequência dos genes dos tipos TEM $(82,2 \%)$, SHV $(67,8 \%)$ e CTX-M $(21,1 \%)$ foi similar a reportada por Mulvey e cols ${ }^{15}$ respectivamente, 77, 67,5 e 28\% em amostras de Eschericia coli e Klebsiella spp.
A alta prevalência de ESBLs do tipo CTX-M em Escherichia coli relatada em estudos internacionais mais recentes ${ }^{79}{ }^{30}$, não foi constatada neste estudo: apenas um isolado de Escherichia coli evidenciou ESBL do tipo CTX-M. Galas e cols ${ }^{9}$ consideraram Escherichia coli armazenando ESBL do tipo CTX-M como a enterobactéria produtora de ESBL mais frequentemente encontrada na França.

Entre as 19 amostras CTX-M positivas, 14 apresentaram maior nível de resistência (menor diâmetro de halo) a cefotaxima do que a ceftazidima. Este achado confirma a afirmação de que a elevada resistência a cefotaxima e a menor resistência a ceftazidima estão associadas a presença de ESBLs do tipo CTX-M 2224.

A ocorrência de mais de um tipo de ESBL em um mesmo isolado é bastante comum, sendo a associação mais frequente a dos tipos TEM e SHV 10121519252729 . Em Escherichia coli e Klebsiella pneumoniae Jeong e cols ${ }^{10}$ encontraram 25 (4,9\%) isolados produzindo essa combinação de ESBLs e Sanguinetti e cols ${ }^{25}$ reportaram $28(5,5 \%)$, em espécies da família Enterobacteriaceae.

Neste estudo, os métodos fenotípicos constataram que $67,8 \%$ dos isolados eram produtores de ESBLs, entretanto, pelo método molecular (PCR) este percentual foi de 93,3\%; a comparação entre os métodos revelou pelo Teste para Proporções Populacionais $\mathrm{p}$ diferenças significativas $(\mathrm{p}<0,0001)$. Por outro lado, empregando-se o teste do qui-quadrado de Independência, demonstrou-se que as ESBLs dos tipos TEM e SHV (detecção molecular) estão associadas aos testes fenotípicos positivos $(\mathrm{p}<0,05)$. Contudo, ao analisarmos esses resultados devemos levar em conta a presença das $\beta$-lactamases TEM-1, TEM-2 e SHV1, também detectadas pelos primers utilizados neste estudo. Além disso, a presença de mecanismos adicionais de resistência, como enzimas do tipo $\mathrm{AmpC}^{1930}$, mudanças nos poros e $\beta$-lactamases do tipo TEM e SHV com reduzida afinidade aos inibidores de $\beta$-lactamases, pode mascarar a inibição do ácido clavulânico resultando em resultados falsos negativos ${ }^{28}$.

Os métodos fenotípicos para a detecção de ESBLs não estão padronizados para todos os microrganismos capazes de armazenar estas enzimas e, clínica e epidemiologicamente, ainda não apresentam resultados ideais. As melhores alternativas são os métodos moleculares de detecção, mas estes, no entanto, ainda são financeiramente pouco viáveis. Estes fatores tornam a detecção da resistência mediada por ESBLs um importante desafio dos laboratórios de microbiologia dos hospitais brasileiros.

\section{REFERÊNCIAS}

1. Agrawal P, Ghosh AN, Kumar S, Basu B, Kapila K. Prevalence of Extended-Spectrum $\beta$-Lactamases among Escherichia coli and Klebsiella pneumoniae Isolates in a Tertiary Care Hospital. The Indian Journal of Pathology and Microbiology 51: 139-142, 2008.

2. Bonnet R, Dutour C, Sampaio JLM, Chanal C, Sirot D, Labia R, Champs C, Sirot J. Novel Cefotaximase (CTX-M-16) with Increased Catalytic Efficiency Due to Substitution Asp-240 $\rightarrow$ Gly. Antimicrobial Agents and Chemotherapy 45: 2269-2275, 2001.

3. Bonnet R. Growing Group of Extended-Spectrum $\beta$-Lactamases: the CTX-M Enzimes. Antimicrobial Agents and Chemotherapy 48: 1-14, 2004. 
4. Carter MW, Oakton KJ, Warner M, Livermore DM. Detection of Extended-Spectrum $\beta$-Lactamases in Klebsiellae with the Oxoid Combination Disk Method. Journal of Clinical Microbiology 38: 4228-4232, 2000.

5. Clinical and Laboratory Standards Institute. Performance Standards for Antimicrobial Disk Susceptibility Tests; Approved Standard-Eighth Edition. CLSI/NCCLS document M2-A8 [ISBN 1-56238-485-6]. Clinical and Laboratory Standards Institute, 940 West Valley Road, Suite 1400, Wayne, Pennsylvania 19087-1898 USA, 2003.

6. Clinical and Laboratory Standards Institute. Performance Standards for Antimicrobial Susceptibility Testing; Fifteenth Informational Supplement. CLSI/ NCCLS document M100-S15 [ISBN 1-56238-556-9]. Clinical and Laboratory Standards Institute, 940 West Valley Road, Suite 1400, Wayne, Pennsylvania 19087-1898, 2005

7. Fang H, Ataker F, Hedin G, Dornbusch K. Molecular Epidemiology of ExtendedSpectrum $\beta$-Lactamases among Escherichia coli Isolates Collected in a Swedish Hospital and Its Associated Health Care Facilities from 2001 to 2006. Journal of Clinical Microbiology 46: 707-712, 2008

8. Freitas ALP, Machado DP, Soares FSC, Barth AL. Extended-Spectrum $\beta$-Lactamases in Klebsiella spp and Escherichia coli Obtained in a Brazilian Teaching Hospital: Detection, Prevalence and Molecular Typing. Brazilian Journal of Microbiology 34: 344-348, 2003

9. Galas M, Decousser J, Breton N, Godard T, Allouch PY, Pina P, Collège de Bactériologie Virologie Hygiène (ColBVH) Study Group. Nationwide Study of the Prevalence, Characteristics, and Molecular Epidemiology of Extended-Spectrum $\beta$-Lactamase-Producing Enterobacterieceae in France. Antimicrobial Agents and Chemotherapy 52: 786-789, 2008.

10. Jeong SH, Bae IK, Lee JH, Sohn SG, Kang GH, Jeon GJ, Kim YH, Jeong BC, Lee SH. Molecular Characterization of Extended-Spectrum $\beta$-Lactamases Produced by Clinical Isolates of Klebsiella pneumoniae and Escherichia coli from a Korean Nationwide Survey. Journal of Clinical Microbiology 42: 2902-2906, 2004.

11. Livermore DM, Canton R, Gniadkowski M, Nordmann P, Rossolini GM, Arlet G, Ayala J, Coque TM, Kern-Zdanowicz I, Luzzaro F, Poirel L, Woodford N. CTX-M Changing the Face of ESBLs in Europe. The Journal of Antimicrobial Chemotherapy 59: $165-174,2007$

12. Luzzaro F, Mezzatesta M, Mugnaioli C, Perilli M, Stefani S, Amicosante G, Rossolini GM, Toniolo A. Trends in Production of Extended-Spectrum $\beta$-Lactamases Among Enterobacteria of Medical Interest: Report of the Second Italian Nationwide Survey. Journal of Clinical Microbiology 44: 1659-1664, 2006.

13. Mugnaioli C, Luzzaro F, De Luca F, Brigante G, Amicosante G, Rossolini GM Dissemination of CTX-M-Type Extended-Spectrum $\beta$-Lactamase Genes to Unusual Hosts. Journal of Clinical Microbiology 43: 4183-4185, 2005.

14. Mugnaioli C, Luzzaro F, De Luca F, Brigante G, Perilli M, Amicosante G, Stefani S, Toniolo A, Rossolini GM. CTX-M-Type Extended-Spectrum $\beta$-Lactamases in Italy: Molecular Epidemiology of an Emerging Countrywide Problem. Antimicrobial Agents and Chemotherapy 50: 2700-2706, 2006.

15. Mulvey MR, Bryce E, Boyd D, Ofner-Agostini M, Christianson S, Simor AE, Paton S, The Canadian Hospital Epidemiology Committee of the Canadian Nosocomial Infection Surveillance Program, Healt Canada. Ambler Class A Extended-Spectrum $\beta$-Lactamase-Producing Escherichia coli and Klebsiella spp. in Canadian Hospitals. Antimicrobial Agents and Chemotherapy 48: 1204-1214, 2004.

16. Nogueira KS, Higuti IH, Nascimento AJ, Terasawa LB, Oliveira S, Matos AP, Souza HAPHM, Cogo LL, Costa LMD. Ocurrence of Extended-Spectrum $\beta$-Lactamases in Enterobacteriaceae Isolated from Hospitalized Patients in Curitiba, Southern Brazil. Brazilian Journal of Infectious Diseases 10: 390-395, 2006.

17. Paterson DL, Bonomo RA. Extended-Spectrum $\beta$-Lactamases: a Clinical Update. Clinical Microbiology Reviews 18: 657-686, 2005.

18. Perez F, Endimiani A, Hujer K, Bonomo RA. The Continuing Challenge of ESBLs. Current Opinion in Pharmacology 7: 459-469, 2007.
19. Perilli M, Dell'Amico E, Segatore B, de Massis MR, Bianchi C, Luzzaro F, Rossolini GM, Toniolo A, Nicoletti G, Amicosante G. Molecular Characterization of Extended-Spectrum $\beta$-Lactamases Produced by Nosocomial Isolates of Enterobacteriaceae from an Italian Nationwide Survey. Journal of Clinical Microbiology 40: 611-614, 2002.

20. Pfaller MA, Segreti J. Overview of the Epidemiological Profile and Laboratory Detection of Extended-Spectrum $\beta$-Lactamases. Clinical Infectious Diseases 42 : S153-163, 2006

21. Pitout JDD, Church DL, Gregson DB, Chow BL, McCracken M, Mulvey MR, Laupland KB. Molecular Epidemiology of CTX-M-Producing Escherichia coli in the Calgary Health Region: Emergence of the CTX-M-15-Producing Isolates. Antimicrobial Agents and Chemotherapy 51: 1281-1286, 2007.

22. Pitout JDD, Hossain A, Hanson ND. Phenotypic and Molecular Detection of CTXM- $\beta$-Lactamases Produced by Escherichia coli and Klebsiella spp. Journal of Clinical Microbiology 42: 5715-5721, 2004

23. Rasheed JK, Jay C, Metchock B, Berkowitz F, Weigel L, Crellin J, Steward C, Hill B, Medeiros AA, Tenover FC. Evolution of Extended-Spectrum $\beta$-Lactam Resistence (SHV-8) in a strain of Escherichia coli during Multiples Episodes of Bacteremia. Antimicrobial Agents and Chemotherapy 41: 647-653, 1997.

24. Rodriguez-Baño J, Navarro MD, Romero L, Martinez-Martínez L, Muniain MA, Perea EJ, Pérez-Cano R, Pascual A. Epidemiology and Clinical Features of Infections Caused by Extended-Spectrum $\beta$-Lactamase-Producing Escherichia coli in Nonhospitalized Patients. Journal of Clinical Microbiology 42: 1089-1094, 2004.

25. Sanguinetti M, Posteraro B, Spanu T, Ciccaglione D, Romano L, Fiori B, Nicoletti G, Zanetti S, Fadda G. Characterization of Clinical Isolates of Enterobacteriaceae from Italy by the BD Phoenix Extended-Spectrum $\beta$-Lactamase Detection Method. Journal of Clinical Microbiology 41: 1463-1468, 2003.

26. Santos DF, Pimenta FC, Alves R, Montalvão ER, Santos DB, Carmo Filho JR Extended-Spectrum $\beta$-Lactamases Producing Klebsiella pneumoniae Isolated in Two Hospitals in Goiânia/Brazil: Detection, Prevalence, Antimicrobial Susceptibility and Molecular Typing. Brazilian Journal of Microbiology 39: 608$612,2008$.

27. Spanu T, Luzzaro F, Perilli M, Amicosante G, Toniolo A, Fadda G. The Italian ESBL Study Group. Occurrence of Extended-Spectrum $\beta$-Lactamases in Italy: Implications for Resistance to $\beta$-Lactams and Other Antimicrobial Drugs. Antimicrobial Agents and Chemotherapy 46: 196-202, 2002.

28. Steward CD, Rasheed JK, Hubert SK, Biddle JW, Raney PM, Anderson GJ, Williams PP, Brittain KL, Oliver A, McGowan Jr JE, Tenover FC. Characterization of Clinical isolates of Klebsiella pneumoniae from 19 Laboratories Using the National Committee for Clinical Laboratory Standards Extended-Spectrum $\beta$-Lactamase Detection Methods. Journal of Clinical Microbiology 39: 2864-2872, 2001.

29. Tasli H, Bahar H. Molecular Characterization of TEM- and SHV-Derived ExtendedSpectrum $\beta$-Lactamases in Hospital-Based Enterobacteriaceae in Turkey. Japanese Journal of Infectios Diseases 58: 162-167, 2005.

30. Tofteland S, Haldorsen B, Dahl KH, Simonsen GS, Steinbakk M, Walsh TR, Sundsfjord A, Norwegian ESBL Study Group. Effects of Phenotype and Genotype on Methods for Detection of Extended-Spectrum $\beta$-Lactamases-Producing Clinical Isolates of Escherichia coli and Klebsiella pneumoniae in Norway. Journal of Clinical Microbiology 45: 199-205, 2007.

31. van Soolingen D, Hermans PWM, de Haas PEW, Soll DR, van Embden JDA. Occurrence and Stability of Insertion Sequences in Mycobacterium tuberculosis Complex Strains: Evaluation of an Insertion Sequence-Dependent DNA Polymorphism as a Tool in the Epidemiology of Tuberculosis. Journal of Clinical Microbiology 29: 2578-2586, 1991

32. Wiegand I, Geiss HK, Mack D, Stürenburg E, Seiferts H. Detection of ExtendedSpectrum $\beta$-Lactamases among Enterobacteriaceae by Use of Semiautomated Microbiology Systems and Manual Detection Procedures. Journal of Clinical Microbiology 45: 1167-1174, 2007. 